In: Deutsches Jugendinstitut (Hrsg.): Handbuch Medienerziehung im Kindergarten. Tejl 1 Pädagogische Grundlagen. Opladen 1994, S.171 185

\title{
Handlungsleitende Themen: Schlüssel zur Bedeutung der bewegten Bilder für Kinder'
}

\section{Auf der Suche nach einem Konzept: "Bedeutung von Medien für Kinder"}

Nachdem Fernsehen Ende der 60er, Anfang der 70er Jahre alltäglich und selbstverständlich geworden war, begann die Diskussion darüber, was denn durch diese ,Veraltäglichung des Fernsehens" (vgl. BachmalR 1990) letztlich passiert ist. Es gab vielfältige Versuche, parallel zur Medienentwicklung (Buch / Film/Fernsehen/Video) und zur Integration der Medien in das Alltagsleben der Menschen, die Beziehung Mensch - Medien zu beschreiben und prägende Strukturen und Abhängigkeiten zu analysieren. Üblich war und ist, gerade auch bei der Medienpädagogik, dabei das Schwergewicht auf die Medien selber zu legen: Wie beeinflussen die Medien die Menschen? Wie lassen sich die Medien für Lernprozesse verwenden? Kann, muß man die Kinder vor Medien bewahren? usw. usw. Von der Fixierung auf die

\footnotetext{
1 Im folgenden werden die Gedankengänge und theoretischen Quellen zusammengefaßt, die mich Ende der 70er Jahre zum Konzept der ,handlungsleitenden Themen" gefiihrt haben. Die entsprechenden Argumentationsschritte finden sich in folgenden Veröffentlichungen: Ben Bachmatr 1979, 1980a, 1980b, 1982 und 1984.
}

Medien bei der Untersuchung und Bewertung der Mensch-Medien-Beziehung wegzukommen, war nicht leicht. Die entscheidende Weiche dafür war, Fernsehen als eine spezifische Form menschlicher Kommunikation zu verstehen. Für die deutschsprachige Medienpädagogik brachte BAACKE (1973a; 1973b) dazu die theoretische und praktische Anleitung auf den Weg.

Ein wichtiger Argumentationsstrang geht dabei auf den sogenannten ,Symbolischen Interaktionismus" zurück, der von G.H. MEAD zu Beginn dieses Jahrhunderts in den USA entwickelt wurde. Thm ging es darum, die spezifisch menschliche Form von Kommunikation zu erläutern, die die bloße Verflechtung von Reizen und antwortenden Reaktionen grundsätzlich hinter sich läßt. So reagieren z.B. zwei Boxer nie nur aufeinander: A schlägt $\mathrm{zu}, \mathrm{B}$ versucht auszuweichen (MEAD 1968, 82). Beide Boxer haben komplizierte Regeln, Absichten und Erfahrungen in ihren „Köpfen“, die ihre Manöver anleiten.

Menschen handeln aufgrund der Bedeutungen, die sie sich im Laufe ihres Lebens mit ihren Erlebnissen und Erfahrungen angeeignet haben (Blumer 1973, $80 \mathrm{ff}$.). Diese 
Aneigung ist nun alles andere als ein mechanischer Prozeß, sondern findet immer in einem komplexen Geflecht von Handlungen, Bedeutungen und Symbolen statt, die sich im Lauf der Zeit verschieben und überlagern. Solche komplexen Geflechte lassen sich laufend im Kindergarten entdecken. Dazu ein Beispiel, bei dem eine Gruppe von fünf Jungen mit Bauklötzchen und Spielfiguren spielt:

Die Jungen bauen eine verschachtelte Burg aus einfachen Holzbauklötzen. Auf der obersten Plattform thront drohend mit aufgerissenem Rachen ein Ungeheuer aus Plastik. Es sieht aus wie ein Drache oder Dinosaurier. Er erinnert an die drohenden Monster aus einschlägigen Filmen mit Urweltungeheuern, wie z.B. Godzilla, oder Riesendinosauriern. Auf der Ebene darunter steht die bunte Plastikfigur eines Mannes. Davor, in der ersten Ebene, in der ein Angriff auf die Burg stattfinden könnte, sind die Turtles postiert. Sie sind eindeutig auf Abwehr ausgerichtet - nicht auf Kampf. Sie haben jedoch ihre Wurf- und Kampfmaschine in Stellung gebracht, ein Katapult, mit dem sich auch tatsächlich eine Schleimmasse - sie wird zusammen mit dem Spielzeug verkauft - abschießen läßt. Mit ernsthafter, aber auch freundlich lustiger Miene, lassen sich die Jungen mit ihrer Spielkonstruktion fotografieren.

Bleibt man nah an einer Kommunikationstheorie, die menschliche Beziehungen von $\mathrm{Be}$ deutungen her aufschlüsselt, ist die Unterscheidung zwischen Sprache (allgemein: Symbolsysteme) und sprechen (allgemein: etwas mit Symbolen darstellen) wichtig (vgl. Saussure 1967). Die jeweilige Sprache einer Kultur ist den Menschen, insbesondere den aufwachsenden Kindern vorgegeben. Sprache ist ein objektiv vorhandenes System von Zeichen und Bedeutungen, aus dem prinzipiell niemand heraus kann. Heute ist Fernsehen ein wichtiger Teil der Sprache unserer Kultur, das Bedeutungen vorgibt. Eine Sprache ohne Menschen bleibt jedoch stumm. Indem die
Menschen sprechen, verwenden sie die vorgegebene Sprache, eignen sich deren Strukturen und Bedeutungen an, verändern sie dabei jedoch immer, indem sie sie in ihrer und auf ihre konkrete Lebenswelt anwenden. Auch mit den Figuren, Bildern und Geschichten des Fernsehens sprechen Kinder heute, indem sie die vorfabrizierten Bedeutungen, z.B. die der hektischen und kämpfenden Figuren der Fernsehserie „Ninja Turtles“ übernehmen. Indem sie diese Figuren in ihre Spielkonstruktion integrieren, entsteht etwas Neues, wiederum etwas Bedeutsames, bedeutsam für diese Kinder und in dieser Spielsituation. Solche Spielkonstruktionen sind für die Kinder von Bedeutung und machen für sie Sinn.

Was macht diesen Sinn aus? Sinn konkretisiert sich in der aktuellen Kindergartensituation mit ihren sozialen Beziehungen und aufgrund der Erfahrungen und Ziele der Kinder. In ihrer Spielkonstruktion verbinden sie die Turtles-Geschichten des Fernsehens mit anderen Erlebnissen, z.B. jenen, die sie von zu Hause in den Kindergarten mitbringen, oder mit dem, was ihnen in ihrer Kindergruppe im Moment wichtig ist. Natürlich verknüpfen die Kinder die ,,mitgebrachten“ Erlebnisse und das, was aktuell ,los“ “ ist, mit dem, was sie spontan wollen und anstreben (MEAD 1968, 160).

Aufgrund ihrer Lebensgeschichte und ihrer Ziele schaffen sich Menschen innerhalb der Sprache ihrer Kultur und innerhalb der aktuellen Situation jeweils ihren subjektiven Sinn neu. Dieser subjektive Sinn ordnet die Erlebnisse und leitet das Handeln und damit auch die subjektive Wahrnehmung der Bilder des Fernsehens (vgl. auch $\rightarrow$ SCHNEIDER Bd.1, $157 \mathrm{ff}$. und Bd.2). Dazu läßt sich folgender Kernsatz formulieren: 
Fernseh-Rezeption, Fernseh-Erlebnisse, Fernseh-Bilder sind als Deutungsmuster oder Handlungsmuster Verständigungsmittel, sinnvoller Bestandteil des Soziallebens, der alltäglichen Ereignisse, der Lebensgestaltung in der Perspektive der individuellen Lebensgeschichte und der subjektiven Themen innerhalb einer Gesellschaft. Sie sind Bestandteil ihrer Kultur und deren spezifischen Ausdrucks- und Aneignungsformen, die in den Fernsehdarstellungen und Nutzungsformen symbolisch erscheinen.

Wenn die Jungen z.B. die Turtles so wichtig finden, daß sie von ihnen mit in den Kindergarten gebracht werden, wenn sie sich zu einer Gruppe ohne Mädchen zusammenschließen und mit gewissem Stolz ihr Spielarrangement herzeigen, dann tun sie etwas für sich Sinnvolles. Was jeweils das ist, was ihren subjektiven Sinn ausmacht, läßt sich weder aus Bedürfnissen (vgl. DRöGE 1979, $116 \mathrm{ff}$.), noch aus den Medien oder angestrebten Zielen herleiten. Es ist eine eigene und subjektive Leistung der Kinder, die sich auf alle diese „Bestimmungsgrößen“ bezieht. Wie die Kinder dies tun, läßt sich am besten dann nachvollziehen, wenn man sich selbst nach den handlungsleitenden Themen der Kinder fragt.

Mit dem Versuch, die handlungsleitenden Themen zu verstehen, nähert man sich dem subjektiven Sinn der Kinder. Wichtig ist es dann, ihn als Thema zu beschreiben, weil sich dadurch das komplexe Gefüge von Alltag, sozialen Beziehungen, Lebenserfahrungen, Medieninhalten sowie Wünschen und Zielen von den Inhalten her in seiner jeweils subjektiven Bedeutung und Funktion erschließen läßt.

\section{Hilfestellungen, um die handlungsleitenden Themen $z u$ verstehen}

Medienerlebnisse sowie die Geschichten und Figuren der Medien ergeben für Kinder jeweils spezifischen Sinn, weil sie in einer zumeist komplexen Beziehung zu den wesentlichen und handlungsleitenden Themen der Kinder stehen. Diese Beziehung kann man verstehen, wenn man die Unterscheidung zwischen „Sprache“ (allgemein: die Symbolsysteme, das symbolische Material einer Kultur, wie Schriftsprache, Umgangssprache und symbolisches Material des Fernsehens) und „Sprechen“ (allgemein: symbolische Aussagen und symbolische Darstellungen einzelner Menschen, z.B. Erzählen, Spielen, Malen) aufgreift. Das symbolische Material einer Kultur, das Kinder in ihrer Lebenswelt als Sprache, Fernsehen, Konsumobjekte usw. vorfinden, beinhaltet Themen der Menschen. ${ }^{2}$ Manche dieser medialen Themen lassen sich leicht entschlüsseln, was - umgangssprachlich - nicht selten mit der „Botschaft eines Films“ beschrieben wird. Andere der in Medien oder

2 In diesem Zusammenhang war bzw. ist das Konzept der „generativen Themen“ von Paulo FREIRE anregend. „Ich muß noch einmal unterstreichen, daß sich das generative Thema nicht im Menschen, abgesehen von der Wirklichkeit, finden läßt, auch nicht in der Wirklichkeit, abgesehen vom Menschen - noch viel weniger in einem ,Niemandsland'. Es ist nur innerhalb des Mensch-WeltVerhältnisses erfaßbar. Wer nach dem generativen Thema sucht, fragt nach dem Denken der Menschen über die Wirklichkeit und nach seinem Handeln in der Wirklichkeit, worin seine Praxis beruht. ... Je aktiver die Menschen im Blick auf die Untersuchung ihrer Themen eingestellt sind, um so mehr vertiefen sie ihre kritische Wahrnehmung der Wirklichkeit und nehmen von ihrer Wirklichkeit Besitz, während sie diese Thematik formulieren“" (FrEIRE 1971, 118). 
sprachlichen Aussagen objektivierten Themen lassen sich nur als Tiefenstruktur erahnen. (Märchen sind ein hervorragendes Übungsfeld, um sich die medialen Themen bewußt zu machen; $\rightarrow 2.1$ ). Zumeist ist es die ,thematische Nähe“" von Filmen, Serien, einzelnen Figuren oder Episoden zu den Kindern, die Sinn für sie ,macht“. Deshalb erinnern sie sich an einzelne Szenen oder Figuren, deswegen spielen sie Szenen oder das Handlungsmuster einer Fernsehserie nach, sprechen darüber mit anderen Kindern, kaufen sie das zur Fernsehserie gehörige Spielzeug. Nicht selten ist es aber auch die soziale Situation, in die die Medien eingebettet sind (z.B. mit einem besonders gruseligen Film vor anderen angeben) oder die Art und Weise der Rezeption (z.B. ohne Anstrengung versorgt werden) oder auch die Dramaturgie eines Films bzw. einer Serie (z.B. hektische Betriebsamkeit), die die Kinder ansprechen, für sie Sinn machen.

„Für sie Sinn machen“ heißt, die rezipierten Medien entsprechen den handlungsleitenden Themen der Kinder $(\rightarrow$ SchMIDBAUER/LÖHR Bd.1, $185 \mathrm{ff}$.). Diese Entsprechung kann leicht erkennbar sein, zumeist ist sie jedoch eher indirekt und muß von den Erwachsenen bewußt rekonstruiert werden. Der subjektive Sinn, den Medienerlebnisse und die Darstellungen in Medien für Kinder machen, läßt sich immer nur über die jeweiligen symbolischen Aussagen und symbolischen Darstellungen der jeweiligen Kinder selber erschließen, also über ihre Erzählungen, Bilder, Spiele usw. Die symbolischen Darstellungen der Kinder sind der Zugang, um handlungsleitende Themen zu verstehen. Wichtig ist hierbei: um die symbolischen Darstellungen der Kinder als Hinweis auf ihre handlungsleitenden Themen $\mathrm{zu}$ interpretieren, muß man sich in die Sinnperspektive der Kinder begeben $(\rightarrow 2.2)$.

\subsection{Märchen: symbolisch verdichtete Erzählungen von den Lebensthemen}

Ein Junge, gerade sechs Jahre alt, bekommt das GRIMMsche Märchen „Die Gänsemagd““ vorgelesen. Das Märchen erzählt von einer Königstochter, die als Braut in ein anderes Königreich reist. Die Mutter stattet sie mit „Gold und Silber, Becher und Kleinodien“" aus, gibt ihr ein sprechendes Pferd mit und hoch symbolisch - ein weißes Läppchen mit drei Tropfen ihres Blutes. Unterwegs verdrängt die Kammerjungfer die Prinzessin, macht sich zur falschen Braut, die wahre Königstochter und Braut wird zur Gänsemagd degradiert. Das sprechende Pferd, dem die Kammerjungfrau den Kopf hat abschlagen lassen, bleibt als sprechender Tröster und Helfer. Der König und Vater des Bräutigams entdeckt die Schönheit der Gänsemagd, unterstützt sie, den Betrug an ihr zur Sprache zu bringen, und verhilft ihr zu ihrem Bräutigam. Die falsche Braut wird auf deren eigene und unbedachte Empfehlung hin auf übelste Weise zu Tode gefoltert.

Nachdem das Märchen vorgelesen ist, will der Junge wissen, wie das mit dem Pferd war, das reden konnte und dessen Kopf abgeschlagen wurde. Nach einer kurzen Erläuterung beschäftigen ihn die Erlebnisse des vergangenen Nachmittags: „,Das heute auf der Kirmes das war schön. Nur ihr seid dauernd so lange bei Geschäften stehen geblieben." Antwort: „Du bist auch dauernd bei Bonbongeschäften und den Karussells stehengeblieben." Danach kommt es zu einem Gespräch über Autoskooter und über den Streit mit der größeren Schwester. Nach einer Pause fragt der Junge 
dann: ,Warum hat die Königstochter ihrer Mutter keinen Brief geschrieben? Die Mutter hätte doch Ritter schicken können und die hätten doch kämpfen können."

In diesem Märchen geht es darum, welche Hürden ein Mädchen überwinden und welche Schritte sie zurückgehen und auch wieder vorwärts gehen muß, um zur Frau zu werden. Dabei spielt auch Reden eine wichtige Rolle; ein abgeschlagener Pferdekopf spricht, sie muß ihr Schicksal auf Umwegen dem Königsvater erzählen. Wichtig ist die Schönheit des Mädchens, an der ihre wahre Identität erkannt wird. Kampf jedoch ist in dieser Phase, eine erwachsene Frau zu werden, unwesentlich. Der Junge kann dies alles nicht verstehen, denn Reden ist für ihn keine Möglichkeit, sich gegenüber der älteren Schwester und den Eltern durchzusetzen. Für ihn ist Kampf angesagt; jedoch in differenzierter Form mit Hilfe starker, kämpfender Männer, die die mächtige Mutter schickt.

Die Handlung des Märchens hat die Erfahrungen vieler Erzähler und Zuhörer aufgesogen, wie denn der Schritt vom Mädchen zur Braut, von der Familie der Kindheit in die Familie des Erwachsenenlebens ablaufen könnte. Die Konflikte und Lösungen dieser Entwicklung werden symbolisch verdichtet, sind aber klar und verständlich, wenn man das Thema des Märchens aufgreift (vgl. zur Interpretation BetTelheIM 1977, $157 \mathrm{ff}$. und 1988, 47). Dies Thema ist dem Jungen jedoch völlig verschlossen. Sein Thema ist, sich gegen eine übermächtige Familie durchzusetzen, und dabei ist ihm männliche, kämpfende Unterstützung wichtig. Die handlungsleitenden Themen organisieren nicht nur die Rezeption und Verarbeitung des Märchens, sondern in vergleichbarer Weise auch die der heute dominanten Medien in der aktuellen Lebenswelt der Kinder, nämlich Fernsehen, Hörspielkassette und Video.

Die für Kinder relevanten Themen kommen im Märchen verschlüsselt zur Sprache. BETTELHEIM hat darauf hingewiesen: ,In den Märchen kommen die schweren inneren Spannungen des Kindes so zum Ausdruck, daß es diese unbewußt versteht; und ohne die heftigen inneren Kämpfe des Heranwachsenden herunterzuspielen, bieten sie Beispiele dafür, wie bedrückende Schwierigkeiten vorübergehend oder dauerhaft gelöst werden können... Das Kind ist ... verzweifelten Gefühlen der Einsamkeit und Absonderung ausgesetzt... Meist kann es diese Empfindungen nicht in Worten ausdrïcken oder doch nur indirekt: Angst vor dem Dunklen, vor einem Tiger, Angst um seinen Körper... Das Märchen ... nimmt diese existentiellen Ängste sehr ernst und spricht sie unmittelbar aus: das Bedürfnis, geliebt zu werden, und die Furcht, als nutzlos zu gelten; die Liebe zum Leben und die Furcht vor dem Tode" (Bettelheim 1983, 12; 17). Hiermit umschreibt Bettelheim wichtige Lebensthemen der Menschen, von denen die Märchen in einer magisch mythischen Weise ${ }^{3}$ erzählen. Seine psychologische Märchen- und Medientheorie „Kinder brauchen Märchen“ (1977) öffnet den Königsweg, um sich als Erwachsener für die Themen der Medien und die handlungsleitenden Themen von Kindern zu sensibilisieren.

3 Vgl. hierzu die kulturhistorischen und wissensphilosophischen Überlegungen von Ernst CASSIRER (1990, $116 \mathrm{ff}$.) zu den symbolischen Formen der Menschen bzw. Kulturen. 


\subsection{Symbolische Darstellungen der Kinder entschlüsseln}

Die modernen Medien haben einen neuen Typ von Kommunikation (medienvermittelte Kommunikation $=$ Medienkommunikation) selbstverständlich gemacht, der wenig mit dem von der Pädagogik favorisierten Kommunikationstyp des Miteinanderredens zu tun hat. Bei der Medienkommunikation stehen sich nicht mehr konkrete Menschen gegenüber, statt dessen ist Kommunikation, wie jede andere Art von Industrieproduktion und Konsum, in deutlich geschiedene Sphären getrennt: in die Welt der Medien (Produktion, Technik und Ökonomie) und in die Lebenswelt der Menschen (Rezeption der Medieninformationen). Zunehmend differenziert sich die Lebenswelt der Menschen gerade auch nach den ihnen wichtigen Medien: Irgendwo und irgendwie werden die Medien produziert, die von bestimmten Adressatengruppen genutzt werden, die sich nach Alter und Lebensstilen differenzieren können. Die Medien liefern die Skripts und Themen für ihre Lebensstile, Szenen und Milieus (vgl. Hengst 1990, 191 ff.).

\section{Das Fremde, d.h. die Medienkommunikation} von Kindern verstehen: In dieser getrennten Welt der Medien und des Alltags ist es nicht leicht, den Sinn zu entdecken, den die Medien für Kinder machen, und dabei den Sinn konsequent aus der Perspektive der Kinder zu entschlüsseln. Hierbei ist eine der Theorien des Handelns in der Industriegesellschaft hilfreich, die Ethnomethodologie (WEINGARTEN u.a. 1976), die von folgenden Argumenten ausgeht. Die verschiedenen und auseinanderfallenden Lebensbereiche der Industriegesellschaft lassen es nicht mehr $\mathrm{zu}$, daß man durch gemeinsames Tun und Handeln in einer gemeinsam geteilten Welt von Bedeutungen lebt. Deshalb geht es z.B. Erwachsenen ähnlich wie den Ethnologen, die in eine fremde Kultur eines anderen Kontinents gehen und diese verstehen wollen. Will man verstehen, was dort passiert, muß man sich in die Sinn- und Handlungsperspektive der beobachteten Fremden begeben. Was Kinder in ihrer, den Erwachsenen fremden Lebens- und Medienwelt tun, sagen, malen, spielen, ist das symbolische Material, das es nach den handlungsleitenden Themen zu befragen und zu deuten gilt. Die Aussagen, Spiele, d.h. die symbolischen Darstellungen der Kinder, sind immer auch Objektivationen des komplexen Prozesses der Sinnorientierung.

Die Bedeutung von Fernsehen für Kinder kann man nur über die symbolischen Darstellungen der Kinder rekonstruieren. Das ist eine Aufgabe, die dem Lösungsweg eines Puzzles ähnelt: Man sieht sich die einzelnen Elemente des Puzzles genau an und fragt sich, welches Bild sie wohl zeigen werden. Die Metapher des Puzzles verweist auf folgendes: Man muß sich a) in die Perspektive des Kindes versetzen, um seine Themen zu entdecken; b) eine genaue Analyse der relevanten Elemente von ,Medienkommunikation" durchführen, um die Sinnperspektive der Kinder tatsächlich einzunehmen. Die relevanten Elemente von „Medienkommunikation" sind:

- die Themen der Kinder. Kinder haben, wie alle Menschen, Ziele, Wünsche, Träume usw., die um bestimmte Themen kreisen: z.B. groß und stark zu sein; geliebt zu werden; in die spannende Welt hinauszugehen und dabei doch beschützt zu werden; als schön oder erfolgreich be- 
wundert zu werden usw. Je nach Lebensgeschichte und Umfeld, in dem die Kinder leben, entstehen bestimmte Themen und verändern sich. Dabei spielt die jeweilige Freundschaftsgruppe der Gleichaltrigen eine prägende Rolle. In unserer Kultur sind zwei gegenläufige Themen vorrangig: Zum einen Themen der $A b$ grenzung und der subjektiven Individualität, wie groß, schnell, dumm, bös, häßlich, schön, erfolgreich bin ich im Vergleich $\mathrm{zu}$ anderen, d.h. konkurrieren. Zum anderen Themen des symbiotischen Dazugehörens, z.B. geliebt, getragen, gefüttert, anerkannt zu werden, d.h. Auflösung und Zerstörung von individuellen Grenzen und Konturen.

- die Bilder, Figuren und Geschichten auf dem Bildschirm. Unabhängig von den $\mathrm{Zu}-$ schauern produziert eine kompliziert verschachtelte und mächtige Medienindustrie Filme bzw. Medien, die aus Bildern, Figuren und Geschichten bestehen. Dazu greifen die Medienmacher in schwer nachzuvollziehender Weise auf die vorhandenen Geschichten, Bilder und Figuren unserer oder anderer Kulturen zurück (z.B. auf die Figur der ,Heidi“, auf uralte Mythen, bekannte Filmgeschichten). Dabei entsteht ein schillerndes Geflecht von Bildern, Figuren und Geschichten, in denen sich bestimmte Figuren und Erzählweisen in den Vordergrund drängen, z.B. „Rambo“ und/oder realistische Gewaltdarstellungen. Die Bilder, Figuren und Geschichten ,wandern" heute schnell vom Bildschirm weg und werden ein Teil der öffentlichen Sprache oder des privaten Gesprächs.

- die Fernseh-Erlebnisse. Zuschauer sind nie passiv, auch wenn sie weitgehend be- wegungslos vor dem Bildschirm sitzen. Sie holen sich diejenigen Geschichten, Figuren und Bilder aus dem Fernsehen oder aus anderen Medien, die ihnen etwas bedeuten und die ihren Themen entsprechen. Die Bilder, Geschichten und Figuren des Fernsehens werden so zu eigenen Erlebnissen. Die Zuschauer verbinden sie dabei mit ihren persönlichen Gefühlen und Themen.

- die Fernseh-Situationen. Vor dem Bildschirm zu sitzen, ist eine Situation von hoher thematischer Bedeutung für Familien und Zuschauergruppen ( $\rightarrow$ RogGE Bd. 1, 497 ff.). Wann das Fernsehgerät eingeschaltet wird, wer in dieser Situation bestimmen darf, wer stört, sind beispielsweise bedeutsame Aspekte des Soziallebens. Daß man sich vor dem Fernsehapparat eng zusammenkuscheln kann, ist für Familien wie für die Freundesclique wichtig. Kinder probieren hierbei auch aus, wie wichtig sie bei Entscheidungen sind, ob und wie sie Aufmerksamkeit oder Zuwendung bekommen ( $\rightarrow$ AufEnANGER Bd.1, $483 \mathrm{ff}$.).

Der folgende Kernsatz liefert das Modell zur Beschreibung der Funktion handlungsleitender Themen im Rahmen der Medienkommunikation: Die Menschen

- mit ihren jeweils spezifisch individuellen, gruppen- und alterstypischen Themen, die das Handeln sinnvoll leiten (handlungsleitende Themen),

- treffen in Situationen auf symbolisches Material ihrer Kultur (Medien und ihre Symbolik),

- mit dessen Hilfe sie sich im jeweiligen Situationskontext orientieren.

- Dazu eignen sich insbesondere Kinder die kulturelle Symbolik der Medien subjektiv 
an und verarbeiten sie thematisch (Erlebnis, Erfahrung, Wahrnehmung),

- um sich selber oder der sozialen Umwelt etwas mitzuteilen (symbolische Darstellung).

Spielarrangements bei Erikson: ERIKSON hat sich im Rahmen seiner Frage nach der ,,Ritualisierung des Alltagslebens während des gesamten Lebenszyklus“ (ERIKSON 1978, 20) auch damit beschäftigt, wie Kinder mittels Baukli en und einigen kleinen Püppchen ihre Themen zum Ausdruck bringen. Er setzte sich mit einem Kind auf den Fußboden und ,,bat es, ,etwas zu bauen' und darüber ,eine Geschichte zu erzählen“" (ebd. 24). Die Kinder ,versenkten sich in eine Aufgabe, die von einem zwingenden Thema und einem bestimmten Stilgefühl beherrscht wird" (ebd. 25). ERIKSON beschreibt und analysiert eine Turmkonstruktion, die ein farbiger Junge baut, die sehr menschenähnlich aussieht, und bei der der Junge an die Stelle des Kopfes der Bauklotzfigur die Puppe eines farbigen Menschen stellt. Aufgrund der Analyse der "Spielkonstruktion“" und der erläuternden Aussagen des Jungen (die dazu erzählte Geschichte) interpretiert ERIKSON dessen ,zentrale(s) Thema“: ,,... die Hoffnung des Jungen, daß es ihm gelingen möge, alle kritischen Punkte seines Daseins mit der Formel der Einheit von Körper und Geist zu bewältigen" (ebd. 28).

Diese von ERIKSON vorgeschlagene Methode der Spielkonstruktion und der sie erläuternden Geschichte ist ideal, weil sie den Darstellungsweisen der Kinder entspricht. Die Jungen, die mit Bauklötzen und Plastikfiguren der Ninja Turtles spielen, öffnen sich für einen außenstehenden Beobachter, der die handlungsleitenden Themen der Kinder, ihre Erlebnisse mit der Fernsehserie der „Ninja Turtles“ zusammen mit den anderen wichtigen Aspekten ihres Lebens rekonstruieren kann.

\subsection{Die Methode der Spielkonstruktion: Die Sendung mit der Maus - ein Beispiel}

Anhand des folgenden Beispieles soll gezeigt werden, wie mit Hilfe der Spielkonstruktion und dem es begleitenden Gespräch das Thema des Kindes erschlossen werden kann. An einem Elternabend hat eine Mutter die Methode kennengelernt, mit Hilfe von Spielkonstruktionen Medienerlebnisse zum Ausdruck zu bringen. Die Mutter bietet ihrer fünfjährigen Tochter Isa Bauklötze an, mit denen sie ihre Lieblingssendung „Die Sendung mit der Maus" baut. Die Fernseherlebnisse sollen während oder nach diesem Bauen verbalisiert werden. Dazu eignen sich auch Fotos einer Spielkonstruktion, die die Chance geben, Tage später assoziativ auf die Fernseherlebnisse und deren Verarbeitung zurückzukommen.

Der Spielverlauf: ${ }^{4}$ Die Mutter holt die Tonne mit Bauklötzen und schlägt Isa vor, ihre Lieblingssendung zu bauen. Am Anfang nimmt Isa wahllos Bausteine. Dann ist sie begeistert bei der Sache und erklärt, was sie baut. Alles dreht sich um die Maus. Es kommen immer mehr Dinge dazu, die für die Maus bestimmt sind. Die Maus bekommt nicht nur eine Familie, sondern auch einen Bauchnabel.

\footnotetext{
4 Dieses Beispiel wurde von Christina Nordmann dokumentiert. Erläuterungen zu den Aussagen sind in Klammern (...) gesetzt. Die medienpädagogische Bearbeitung von Fernseherlebnissen mittels Spielkonstruktionen fand im Rahmen eines Elternsgesprächskreises statt.
} 
(Die Mutter schaut sich das Gebilde an, das Isa baut.)

Mutter: Das ist keine Kirche, was kann das sonst sein? Hmh, ein Stuhl, nein?

Isa: Ein Haus.

Mutter: Ein Haus, das sieht ja schön aus.

Isa: $\quad$ Siehst du denn das Dach nicht? (...) Drei Dächer.

Mutter: Und wohnt da wer in dem Haus?

Isa: Die Maus. (...) Die Maus hat ein großes Haus.

Isa hat neben das Haus mit den drei Dächern, das sie aus Bauklötzen gebaut hat, ebenfalls aus Bauklötzen eine Figur mit Kopf, Armen, Beinen gelegt. Einen Stein hat sie in die Mitte des Bauchs gelegt. Die Maus hat eine Familie.

Isa: Das da ist die Maus.

Mutter: Wo ist die Maus? Oh, die ist ja toll, Isa.

Isa: Ferlig.

Mutter: Die Maus ist fertig? Die gefallt mir ganz toll. Muß ich ja mal gucken. Die ist ja gar nicht so klein? (...) Isa, und in dem Haus wohnt die Maus?

Isa: Ja.

Mutter: Wohnt da noch wer drin?

Isa: Baby Maus, Vater Maus, Kind Maus und das da ist die Mutter.

Mutter: Das ist die Mutter, willst du die anderen Mäuse auch noch bauen?

Isa: $\mathbf{M m m}$.

Isa beläßt es bei der einen Maus. Sie baut einen Turm für die Baby-Maus.

Mutter: Dann fang mal an, die Maus hat ja ein wunderschönes Haus.

Isa: Jetzt bau ich erst noch den Turm von der Maus.

Mutter: Braucht die einen Turm? Wofür?

Isa: Damit das Baby nicht rauskrabbeln kann. (...) Mama, hier sind die Augen von der Maus.

Mutter: Oh Isa, die ist schön.

Isa: Schön gebaut, ne, ja wußte gar nicht, was ich vorher nehmen sollte.

(...)
Mutter: Du, man erkennt aber trotzdem den Mund, find ich.

Isa: Der Bauchnabel.

Mutter: Oh, der ist wichtig, kann man gut sehen.

(...)

Isa: Da können sie immer rausgehen, da ist die Tuir oben.

Mutter: Ach die Tür, die habe ich ganz vergessen.

Isa: Das ist die Terrassentür und das ist die vordere Tür.

(...)

Isa betont den Bauchnabel der Maus, geht aber auch wieder anf das Haus der Maus und dessen Mobiliar ein. Sie will, daß die Mutter die Maus fotografiert. Damit bekommt ihre Maus und ihr Spielarrangement besondere Bedeutung.

Isa: Fotografiere noch mal die Maus.

Mutter: Soll ich die Maus noch mal fotografieren?

Isa: $\quad \mathrm{Mm}$.

Mutter: Bist du fertig mit Bauen, oder soll noch was dazu kommen?

Isa: Ich bau noch was.

Mutter: Und was wird das?

Isa: Das wird von der Maus... ahm..

Mutter: Mußst einen anderen nehmen.

Isa: Das wird von der Maus... ahm, der Sessel. Mutter: Der Sessel?

Isa: Der Sessel. Und das ist eine Matratze, damit es nicht so hart wird. Ja, für die Maus, wenn sie Purzelbäume macht, braucht sie auch Matratzen, ne. Drei Matratzen, doch zwei, das wird ein kleines Haus.

Interpretation: Es geht mir gut und ich entdecke mich in meiner Welt! Spielarrangement und Gespräch helfen, Aspekte von Isas handlungsleitendem Thema zu erschließen. Eine Feinanalyse der Aussagen und des Spielarrangements sowie der Vergleich mit Fernsehserie bzw. Fernsehfiguren sind die Basis für eine komplexe Beschreibung der jeweiligen handlungsleitenden Themen.

In diesem Fall ist die Grundstimmung der Sendung wichtig: sich die spannende Welt 
anschauen. Das ist möglich, weil man sich aus der Geborgenheit in die weite und aufregende Welt der Dinge, Geschichten und Phantasien begibt. Für Isa ist diese spannende Welt im Augenblick sie selber in ihrer sozialen Umgebung. Sie hat ihre Welt - das ist die Welt ihrer Familie (Haus und Familie der Maus) - entdeckt und untersucht, wie sie aufgebaut ist. Dabei kommt es ihr auf den Mittelpunkt - den Bauchnabel - der Maus an. Thre Mausfigur hat nicht nur Kopf, Bauch, Arme und Beine, also alles, was ein Kind differenzierend von Menschen wahrnimmt, sondern auch einen Mittelpunkt. Diesen Mittelpunkt hat jeder Mensch, ob klein oder groß. Und dieser Mittelpunkt hat etwas damit zu tun, woher die Menschen kommen. Da die Maus, die sie aus Bauklötzen gelegt und deren Mittelpunkt sie mit einem Baustein markiert hat, für alle $\mathrm{Fa}$ milienmitglieder der Maus steht, beschäftigt sie sich auch mit deren Mittelpunkt.

Mit der Maus, die als Fernsehbild keinen Bauchnabel hat, erkundet sie eigenständig, wie die Menschen und wie sie selber als komplette Menschen mit je eigenem Mittelpunkt aufgebaut sind. Sie kann dies ohne Risiko wagen, weil sie die Geborgenheit des Hauses der Maus natürlich nicht nur in der Sendung gesehen hat, sondern diese Geborgenheit im eigenen und sehr differenzierten Familien-Haus erlebt.

\section{Warum weint ein durchsetzungsfähiger Junge beim Fernsehen?}

Im folgenden wird das Fallbeispiel vorgetragen, zu dessen Analyse das erste Mal das Konzept der handlungsleitenden Themen verwendet wurde (BACHMAIR 1989).
In einem Kindergarten schaut eine Gruppe vier-bis sechsjähriger Jungen und Mädchen zusammen mit einem Erzieher und zeitweise einer Erzieherin einmal wöchentlich eine Sendung aus dem ZDF-Kinderprogramm per Video an. Diesmal wird der Film „Ein Tier ist ein Tier“" aus der Serie „Neues aus Uhlenbusch" vorgeführt. Der Film zeigt folgende Geschichte: Ein Junge bekommt ein kleines Schwein geschenkt, das er mit der Flasche aufzieht. Als das Schwein älter wird, steht er vor der Entscheidung, es entweder zu verkaufen, um sich aus dem Erlös ein Fahrrad zu kaufen, oder es zu behalten, weil er das Schwein gern hat. Er entscheidet sich, das Schwein zu verkaufen. Am Schluß der Sendung erhält der Junge ein neues Ferkel geschenkt.

Rezeptionsverlauf: Vor der Sendung kommandiert der sechsjährige David die Kinder der Gruppe herum. Während der Sendung wird die Handlung von allen Kindern spontan kommentiert. David beginnt plötzlich zu weinen, als im Film der Junge, der das Ferkel aufgezogen hat, sagt: „Aber ich kann dich doch nicht behalten. Schweine behält man eben nicht für immer. Ist doch klar. Du wirst ja nicht geschlachtet. Erstmal kommst du zu deinen Geschwistern, und dann kriegst du 'n Haufen Tolles zu essen, bis du alt bist. Dann wirst du erst geschlachtet. Ich kann dich einfach nicht behalten. Ehrlich. Aber warum hab ich dich auf einmal so gern? Das verstehe ich nicht."

Dazu das Wortprotokoll, das zeigt, wie David die Situation zur Regression nutzt:

Michel: Jetzt holt er sich Stroh. Muß er aber weit David schnieft. Er steht auf, dreht sich zum Erzieher, der hinter ihm sitzt und beginnt zu weinen. Er schluckt heftig und bringt keinen artikulierten Ton heraus.

Erzieher: Was ist denn los? David, komm mal her. David, was ist denn los? Sag mal. 
David weint laut.

Das Gerät wird abgeschaltet.

Erzieher: Aber, warum bist du denn so traurig?

David weint weiter.

Erzieher: Weil der Junge jetzt das Schweinchen weggibt?

David (lauter weinend): Jaaa.

Erzieher: Oder hat dich der Holger geärgert?

David: Nee. (Weint, stammelt etwas Unverständliches in einer Art Babysprache.)

Erzieher: Dut willst nicht, daß der Junge das Schwein verkauft.

David (wiederum weinend, kaum verständlich): Ja.

Erzieher: Der Junge hat ja eben auch gesagt, daß er es nicht mehr behalten kann, weil das Schwein größer und größer wird. Ja, und dann kann er es nicht mehr füttern. Der Junge hat ja auch gesagt, daß das Schwein jetzt noch nicht geschlachtet wird, erst wenn es älter ist, als Schwein.

Das Gerät wird wieder angeschaltet.

Die Sendung geht zu Ende, nachdem der Junge ein anderes junges Schwein bekommen hat. David sitzt ganz still und kaut am Daumen. Der Erzieher versucht über Schweine und deren Schicksal zu reden. Er fragt die Kinder, ob sie ebenfalls das zweite Schwein nehmen würden. David bleibt still, auch als der Erzieher ihn persönlich anspricht. Erzieher und die hinzukommende Erzieherin versuchen, dem Gespräch und damit auch David Realitätsbezug zu geben. (.,Du hast doch auch 'nen Kaninchen, David.") Die anderen Kinder gehen dabei auf das Filmmotiv „Tiere schlachten“ ein:

Kind: Ich krieg' auch bald 'nen Kaninchen. Katrin: Ich wiör' das Schweinchen auch haben wollen. Weipt du, wartm? Wenn's groß ist und alt ist, dann schlachte ich das.

Erzieherin: Meinst du, du könntest das auch schlachten?

Katrin: $J a$.

Der Erzieher geht wieder auf den schweigenden David ein, immer noch mit dem gleichen Ziel, von der Filmgeschichte weg zur Realität zu kommen, um danach David von den Regressionen weg zum Realitätscharakter der Filmgeschichte zu führen. Es ge- lingt auch. David stellt heraus, daß er im Gegensatz zur Hauptfigur des Films keine Entscheidungsprobleme zwischen Tier und Spielgerät hat: ,Ich hab, aber einen Hasen und mein Kettcar... Das hab' ich mir nämlich selber gekauft."

Dazu das Tonbandprotokoll:

Erzieher: David, wenn du jetzt auf dem Dorf wohnst, (...), dann kannst du dir auch mal beim Bauern diese kleinen Ferkel angucken.

David weint.

Kind: $\quad$ Ich krieg auch bald so 'n Schweinchen, so 'n Hasen wie David.

Die beiden Erwachsenen reden mit David. Nach einiger Zeit:

David (weinend, man versteht nur): Warum verkauft?

Erzieher: Warum er das verkauft hat? Einmal, weil das ja größer und größer gewachsen ist und weil das jetzt auf ' $n$ andern Bauernhof kommt und da von 'nem Bauern gefuittert wird. Und zweitens, weil er doch das Geld brauchte für sein neues Fahrrad. Und außerdem hat er ja jetzt wieder zum Schluß ein ganz kleines Schweinchen gekriegt, was er großziehen konnte.

David (weinend): Ich fand das eine schöner.

Erzieher: Du fand'st das erste schöner?

David (weinend): Jaaa.

Erzieher: Guck mal. Der Junge hatte sich das ja auch ganz schön lange überlegt. Und er war auch ganz traurig, als er es weggegeben hat.

Erzieherin: Stimmt. Traurig war er auch. Der hatte das Schweinchen auch lieb gehabt.

Erzieher: Guck mal. Zwei Sachen konnte er nicht haben. Das Schweinchen und das Fahrrad. Da mußte er sich für eine Sache entscheiden. Das ist eben auch ganz schön schwer.

David (wütend): Ich hab aber 'nen Hasen und mein Kettcar.

Michel (ungläubig): Hasen und Kettcar?

David: Dashabichmirnämlich selber gekauft.

Erzieher: Das Kettcar? 
David (weinend): $J a$.

Erzieherin: Da hast du aber ganz schön viel Geld gehabt. Der Junge hat nicht soviel Geld gehabt wie du.

David: $\quad$ Ich hätte das Schweinchen nicht an den Bauern verkauft. Ich hätte mir das Schweinchen behalten.

Erzieher: Bis es ganz, ganz groß geworden wäre? David: $J a$.

Nachdem David das Filmmotiv ,Schweinchen verkaufen" realistisch behandelt hat, greift Michel ein anderes, implizites Filmmotiv auf: Schlachten. Er weitet es unrealistisch zu einer aggressiven Größenphantasie aus. Das aggressive Motiv in der Situation, in der David den Realitätsbezug des Erziehers akzeptiert hat, führt dazu, daß die Erzieher sich im Gespräch gegen Michel wenden und sich auf Davids Seite stellen.

Michel: Und dann hätt' ich's verkauft, wenn's groß wäre.

David: Ich aber nicht.

Michel: Dann hätt' ich's geschlachtet.

David: Ich hätt' es nicht geschlachtet. Bis es gestorben wär, hätt' ich's dann behalten.

Michel: $\quad$ Damn hätt' ich die Haut abgerissen und - klatsch - in die Pfanne geschmissen.

Erzieherin: Du hättest es gleich aufgegessen?

Erzieher: Ach Michel, das glaub ich auch nicht. Wenn du erst mal so lange 'nen Schwein hast und das selber großgezogen hast, dann hättest du das auch lieb gehabt. Ich glaub' nicht, daß du das einfach geschlachtet hättest.

Offensichtlich hat die Beziehung zu den beiden Erwachsenen David so ermutigt, daß er mit Holger, dem starken Jungen der Kindergruppe, zu konkurrieren beginnt und dabei auftumpft: , ,(..) hol' ich mir ein so ein Kleines und dann habt ihr keins. (...) Kauf ich mir auch noch so'n Adler (...) und da werdet ihr aufgefressen. "Damit ist der Junge bei aggressiven Motiven angelangt, bei denen es um Größe, Kampf und Macht geht.

Erzieher: David, wirkönnen ja jetzt zusammen mal 'nen ganz kleines Schweinchen malen.
David (unterbricht Holger): Ja, und warum machste immer alles nach?

Erzieher: Was?

David (nölend): Warum macht der Holger mir immer alles nach?

Erzieher: Warum dir Holger alles nachmacht? Was hat der denn nachgemacht?

David: Hatnachgemacht... (Es folgt unverständliches Gestammle.) Wie ich gemacht hatt'; alles macht der Holger immernach.

Erzieher: Habt ihr denn alle Lust, so 'n kleines Schweinchen zu malen?

David (ärgerlich weinend): Ich hätte lieber ein echtes Schweinchen. Wenn ich beim Bauern wohne, hole ich mir so $n$ kleines und dann habt ihr keins.

Erzieher: Das können wir uns dann doch auch mal angucken?

David: $\quad$ Mmh. Das würde größer und wird größer und dann kauf' ich mir auch noch so ' $n$ Adler. Und der hebt euch dann hoch und dann fliegt ihr.

Erzieher: Wir können dann mit dem Adler wegfliegen?

David: $\quad J a$, der fliegt dann mit euch in die Berge und da werdet ihr aufgefressen.

Interpretation: Bei der Filmbesichtigung bricht vermutlich Davids ambivalentes Thema „Bedrohtsein, Zerstörtwerden, Mächtigsein, Angreifen" auf. Die Ambivalenz zeigt sich in seiner gruppendynamischen Aktivität, u.a. in seiner Bemerkung am Ende ,,da werdet ihr aufgefressen" und in seiner heftigen Reaktion auf das Filmmotiv vom Schwein, das zugunsten eines Fahrrades verkauft wird. Die Erwachsenen bieten David eine Situation, in der er direkt agieren und regressiv Bedrohungsängste bearbeiten kann. Das ist die Voraussetzung, von der aus er das Angebot, das Filmmotiv mit seiner Lebenserfahrung in Verbindung zu setzen, aufgreifen kann (Realitätsbezug). Die Zuwendung an David aktiviert jedoch das Langzeit-Thema der Kindergruppe: Konkur- 
renz um die Zuwendung der Erwachsenen und Rivalität um den Platz in der Gruppenhierarchie. Dazu knüpfen Michel wie David bei der aggressiven Seite des Filmmotivs „Schwein an Metzger verkaufen“ an.

Dieser Verlauf der Filmrezeption zeigt die Dominanz der handlungsleitenden Themen, die jedoch eine dafür offene Situation (Regressionsmöglichkeit, Herstellen des Realitätsbezugs durch Erzieher) und entsprechende Filmsymbolik braucht. Zu vermuten ist, daß das Filmmotiv ,,Schwein verkaufen“ gerade im Rahmen der für ,Neues aus Uhlenbusch" typischen, sehr ruhigen, einfühlsamen Dramaturgie seine thematische Funktion aus zwei Gründen entfalten kann. Einmal, weil sie Zeit für subjektive Assoziationen und Phantasien läßt. Zum zweiten, weil sie im Prinzip offen ist für Empathie und Regression. Die spontane pädagogische Strategie der Erwachsenen (Regressionsmöglichkeiten und Realitätsbezug anbieten, aggressive Größensymbolik akzeptieren) zeigt, daß sie angemessen und zumindest für David unter thematischen Gesichtspunkten - hilfreich ist.

\section{Die Erschließungsfunktion handlungsleitender Themen}

Der hier skizzierte theoretische und praktische Weg führt über die symbolischen Äußerungen der Kinder (z.B. Sprachbilder, spontane Aussagen, Spielkonstruktionen) und deren Analyse $\mathrm{zu}$ den handlungsleitenden Themen der Kinder. Das Konizept der handlungsleitenden Themen hilft, die Subjektivität der Kinder zu erschließen, die das komplexe Gefüge des Alltags, der aktuellen sozialen Beziehung und der eigenen Lebens- geschichte sinnbezogen strukturiert. Gelingt es, diese persönlichen und leitenden Themen zu verstehen, erschließt sich für den Erwachsenen die Dynamik, wie sich ein Kind in seiner sozialen und dinglichen Welt, in der heute die Medien eine zentrale Funktion haben, zurechtfindet und wie es dabei die Symbolik der Medien und die Medienerlebnisse nutzt und verarbeitet.

\section{Literatur}

BAACKE, D.: Kommunikation und Kompetenz. Grundlegung einer Didaktik der Kommunikation und ihrer Medien. München 1973a

BAACKE, D. (Hrsg.): Mediendidaktische Modelle: Fernsehen. München 1973b

Bachmair, B.: Alltag ist Gegenstand von Fernschforschung. In: Charlton, M./Bachmair, B. (Hrsg.): Medienkommunikation im Alltag. Interpretative Studien zum Medienhandeln von Kindern und Jugendlichen. München 1990, 57-75

BACHMair, B.: Thematisch und situativ integrierte Fernsehrezeption im Kindergarten. In: ERLINGER, H.-D. (Hrsg.): Kinderfernsehen II. Essen 1989, 35-54

BACHMAIR, B.: Symbolische Verarbeitung von Fernseherlebnissen in assoziativen Freiräumen. Teil 1: Fernsehspuren im Handeln von Kindern. Kassel 1984

Bachimair, B.: Alltagsthemen und Fernseherlebnisse Wie Grundschulkinder Fernseherlebnisse bearbeiten. In: Praxis Schulfernsehen 7. Jg., 1982, H.67, 4-10

BACHMAiR, B.: Technologische Veränderung von Kommunikation als Herausforderung für die Pädagogik. In: SCHÄFER, G./LOCH, W. (Hrsg.): Kommunikative Grundlagen des naturwissenschafilichen Unterrichts. Weinheim 1980a, 147-189

BaChmair, B.: Mit eigenen Augen sehen - der Versuch, Fernsehen didaktisch zu zähmen. In: medien + erziehung 1980b, H.4, 194-204

BACHMAIR, B.: Ethnomethodologie als handlungstheoretische Grundlage einer Didaktik der Kommunikation. In: Bildung und Erziehung 1979, H.3, 229-240

Bettelheim, B.: Brauchen Kinder Fernsehen? In: TelevIZIon 1988, H.1, 4-7

BetTelheim, B.: Kinder brauchen Märchen. Stuttgart 1977

Blumer, H.: Der methodologische Standort des Symbolischen Interaktionismus. In: ARBEITSGRUPPE BIELEFELDER SOzlologen (Hrsg.): Alltagswissen, Interaktion 
und gesellschaftliche Wirklichkeit. Bd. 1. Symbolischer Interaktionismus und Ethnomethodologie. Reinbek 1973, 80-146

CAssirer, E.: Versuch über den Menschen. Einführung in eine Philosophie der Kultur. Frankfurt/M. 1990 (englisches Original 1944)

Dröge, F: Vom Primat der Bedürfnisse im menschlichen Handeln. In: RÜDEN, P.v. (Hrsg.): Unterhaltungsmedium Fernsehen. München 1979, 116-142

ERIKSON, H.: Kinderspiel und politische Phantasie. Stufen in der Ritualisierung der Realität. Frankfurt/M. 1978

Freire, P.: Pädagogik der Untęrdrückten. Stuttgart 1971
Hengst, H.: Szenenwechsel - Die Scripts der Medienindustrie in der Kinderkultur. In: CharltoN, M. / BACHMAIR, B. (Hrsg.): Medienkommunikation im Alltag. Interpretative Studien zum Medienhandein von Kindern und Jugendlichen. München 1990, 191-209

MEAD, G. H.: Geist, Identität und Gesellschaft - aus der Sicht des Sozialbehaviorismus. Frankfurt/M. 1968

SAUSSURE, F. de: Grundlagen der allgemeinen Sprachwissenschaft. Berlin 1967

Weingarten, E./SACK, F./Schenkein, J. (Hrsg.): Ethnomethodologie. Beiträge zu einer Soziologie des Alltagshandelns. Frankfurt/M. 1976 